\title{
Synthesis and reactions of a new 1,1-disubstituted cyclopentadiene
}

\author{
Pelayo Camps* and Tània Gómez \\ Laboratori de Química Farmacèutica (Unitat Associada al CSIC), Facultat de Farmacia, \\ Universitat de Barcelona, Av. Diagonal 643, E-08028, Barcelona, Spain \\ E-mail: camps@ub.edu
}

Dedicated to Prof. Julio Alvarez-Builla on the occasion of his $65^{\text {th }}$ anniversary

\begin{abstract}
The synthesis and several synthetic transformations of methyl 1-benzylcyclopenta-2,4-diene-1carboxylate are described.
\end{abstract}

Keywords: Methyl 1-benzylcyclopenta-2,4-diene-1-carboxylate, norbornenes, norbornadienes, Diels-Alder reaction, (2-iodoethynyl)(phenyl)iodonium triflate

\section{Introduction}

For several years, we have been working on the generation, trapping and dimerization of highly pyramidalized alkenes containing the skeleton of tricyclo[3.3.0.0 $0^{3,7}$ oct-1(5)-ene, as very reactive species for the fast elaboration of complex polycyclic compounds. ${ }^{1}$ These pyramidalized alkenes are usually generated by deiodination of double bridgehead 1,2-diiodo precursors with molten sodium in boiling 1,4-dioxane or $t$-BuLi in THF. In connection with this work, we planned the preparation of a conveniently functionalized 1,1-disubstituted cyclopenta-2,4-diene, to study different model transformations, specially a simple introduction of a 1,2-diiodoethylene functionality.

\section{Results and Discussions}

In this study, we chose methyl 1-benzylcyclopenta-2,4-diene-1-carboxylate 5 as the 1,1disubstituted cyclopenta-2,4-diene, whose ester function opens the way for different transformations, apart from those derived from the presence of the diene substructure. Diene $\mathbf{5}$ was obtained as shown in Scheme 1 from methyl 1-benzyl-2-oxocyclopentanecarboxylate $\mathbf{1}^{2}$ Following a procedure described for a related case, ${ }^{3}$ keto ester $\mathbf{1}$ was reacted with trimethylsilyl 
triflate to give the corresponding silylated enol ether $\mathbf{2}$, which was directly oxidized by bubbling oxygen through a vigorously stirred DMSO solution in the presence of $\mathrm{Pd}(\mathrm{OAc})_{2}$ as the catalyst to give the known ${ }^{4}$ enone 3 in $82 \%$ yield of chromatographed product. $\mathrm{NaBH}_{4}$ reduction of $\mathbf{3}$ in the presence of $\mathrm{CeCl}_{3} \cdot 7 \mathrm{H}_{2} \mathrm{O}$ following the Luche procedure, ${ }^{3,5}$ gave the known ${ }^{4}$ allylic alcohol $\mathbf{4}$, which was subjected as such to acid catalyzed dehydration to give cyclopentadiene 5 in $66 \%$ yield of chromatographed product (Scheme 1).

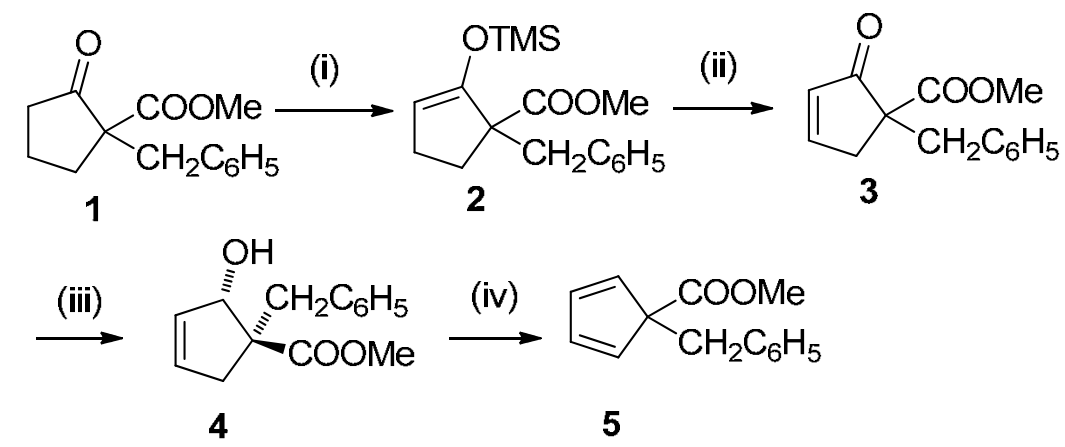

Scheme 1. Preparation of methyl 1-benzylcyclopenta-2,4-diene-1-carboxylate 5. (i) $\mathrm{CF}_{3} \mathrm{SO}_{3} \mathrm{SiMe}_{3}, \mathrm{Et}_{3} \mathrm{~N}, \mathrm{CH}_{2} \mathrm{Cl}_{2}$, rt, 30 min. (ii) $\mathrm{Pd}(\mathrm{OAc})_{2}, \mathrm{O}_{2}, \mathrm{DMSO}$, rt, $24 \mathrm{~h}, 3$ (82\% from 1). (iii) $\mathrm{NaBH}_{4}, \mathrm{CeCl}_{3} \cdot 7 \mathrm{H}_{2} \mathrm{O}, \mathrm{MeOH}$, rt, 1 h. (iv) $p-\mathrm{TsOH} \cdot \mathrm{H}_{2} \mathrm{O}$, benzene, reflux, 18 h, 5 (66 \% from 3).

Diene 5 is a relatively stable compound that slowly dimerizes at room temperature to give after 4 months a unique stereoisomeric dimer 6 in 58\% yield (Scheme 2), still remaining some diene 5. The structure and relative configuration of this dimer were fully established through NMR data: ${ }^{1} \mathrm{H} /{ }^{1} \mathrm{H}$ homocorrelation (COSY and NOESY) and ${ }^{1} \mathrm{H} /{ }^{13} \mathrm{C}$ heterocorrelation experiments $\left({ }^{1} \mathrm{H} /{ }^{13} \mathrm{C}\right.$ gHSQC and gHMBC sequences). Formation of this stereoisomer requires approaching of both components from the side of the less bulky methoxycarbonyl substituent. The stereochemistry of $\mathbf{6}$ coincides with that established by other means for the dimer obtained from a related compound, methyl 1-methylcyclopenta-2,4-diene-1-carboxylate. ${ }^{6}$

Diene 5 participated without problems in standard Diels-Alder reactions. Thus, reaction of crude diene $\mathbf{5}$ with maleic anhydride or cis-1,2-bis-(phenylsulfonyl)ethylene ${ }^{7}$ gave the corresponding endo-adducts 7 and $\mathbf{8}$, respectively (Scheme 2). In both cases, only endo-adducts derived from the addition of the dienophile to the diene from the side of the less bulky methoxycarbonyl group were detected. Formation of the exo-adducts must be disfavored by the steric interaction among the 7-syn substituent and the 2-exo and 3-exo substituents. This type of interaction must be the responsible for the fact that no reaction took place among diene $\mathbf{5}$ and trans-1,2-bis-(phenylsulfonyl)ethylene, after $60 \mathrm{~h}$ in refluxing toluene. In the corresponding adduct, one of the phenylsulfonyl groups will be in an exo-position, thus being very close to the syn-substituent at position 7.

Cis-1,2-Bis(phenylsulfonyl)ethylene has been used as an acetylene equivalent in Diels-Alder reactions, ${ }^{7}$ when combined with the reductive desulfonylation of the cycloadduct with $2 \%$ 
sodium amalgam. This reduction is usually performed in $\mathrm{MeOH}$ in the presence of monosodium phosphate. When we reacted adduct $\mathbf{8}$ with $2 \%$ sodium amalgam under the above conditions, we could isolate slightly impure cyclopropanated compound $\mathbf{1 1}$ and a mixture of $\mathbf{1 1}$ and the expected diene 10 in low yields. Since compound $\mathbf{1 1}$ contains two hydrogen atoms more than diene 10, we considered that these hydrogen atoms must come from the protic medium. When the above reaction was carried out in an aprotic solvent (1,4-dioxane) in the absence of any hydrogen source, diene $\mathbf{1 0}$ was isolated in 53\% yield (Scheme 2). Diene $\mathbf{1 0}$ could not be obtained from anhydride 7 by hydrolysis followed by reaction with $\mathrm{Pb}(\mathrm{OAc})_{4}$, anhydride 7 being the only product recovered in the last reaction.

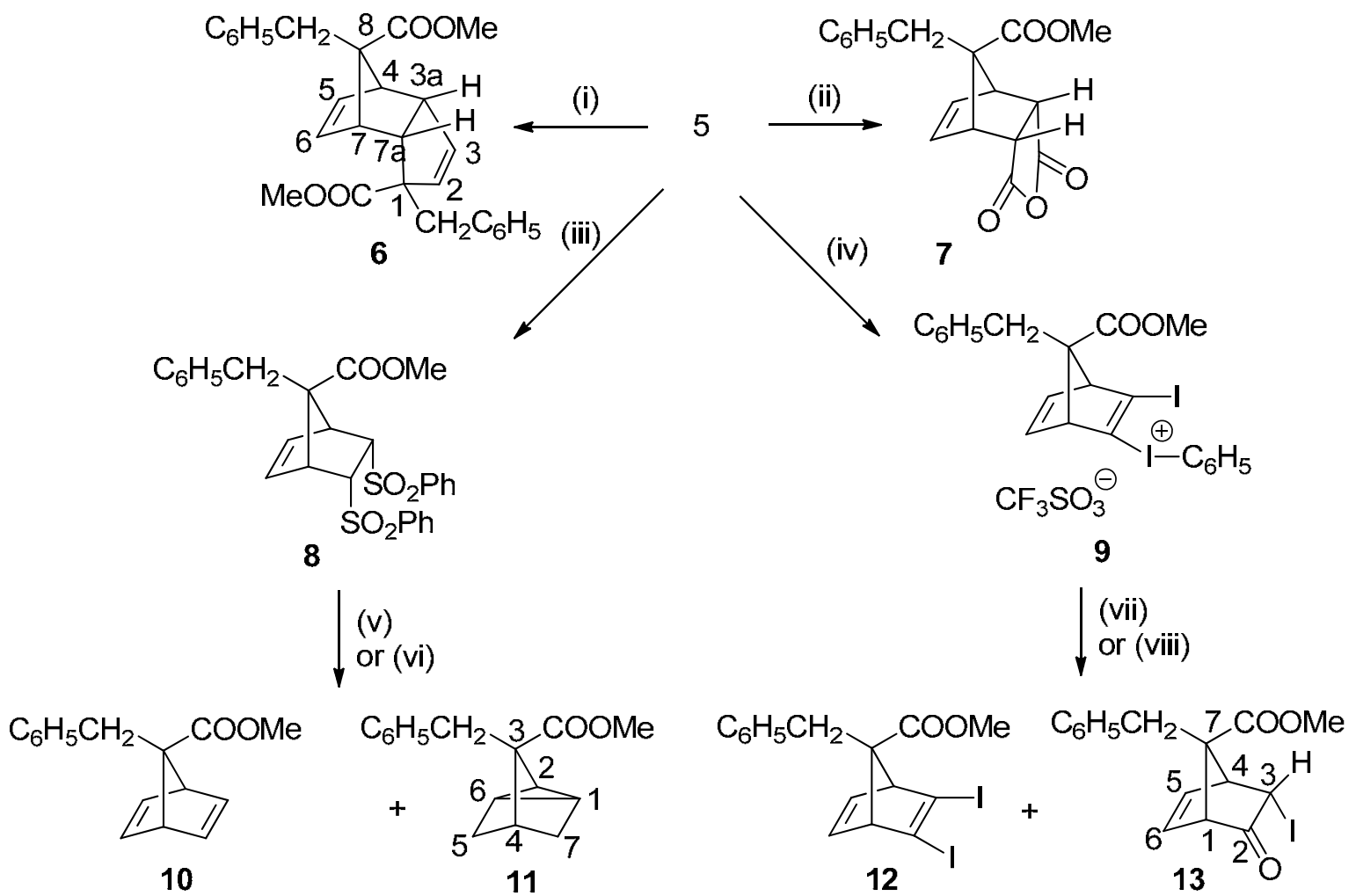

Scheme 2. Reactions from methyl 1-benzylcyclopenta-2,4-diene-1-carboxylate 5. (i) Rt, 120 d, 6 (58\%). (ii) Maleic anhydride, toluene, reflux, $4 \mathrm{~h}, 7$ (90\% from 3). (iii) cis-1,2bis(phenylsulfonyl)ethylene, toluene, $100{ }^{\circ} \mathrm{C}, 15 \mathrm{~h}, 8$ (61\% from 3). (iv) (2-iodoethynyl) (phenyl)iodonium triflate (14), MeCN, rt, 20 h, 9 (79\% from 3). (v) $\mathrm{Na}(\mathrm{Hg})$ 2\%, 1,4-dioxane, rt, 18 h, 10 (53\%). (vi) $\mathrm{Na}(\mathrm{Hg}) 2 \%, \mathrm{NaH}_{2} \mathrm{PO}_{4} \cdot 2 \mathrm{H}_{2} \mathrm{O}, \mathrm{MeOH}, \mathrm{rt}, 24 \mathrm{~h}, 11$ (about 25\%), mixture 10 and 11 (about 35\%). (vii) NaI, CuI, MeCN, $-40{ }^{\circ} \mathrm{C}$ to rt, overnight, 12 (46\%). (viii) Aqueous $\mathrm{NaOH}, \mathrm{CH}_{2} \mathrm{Cl}_{2}$, rt, 18 h, 12 (35\%), 13 (23\%). 


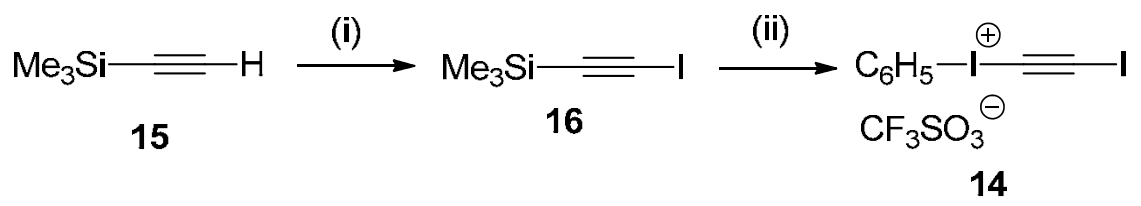

Scheme 3. Preparation of (2-iodoethynyl)(phenyl)iodonium triflate 14. (i) (a) n-BuLi, THF, -78 ${ }^{\circ} \mathrm{C}$, (b) $\mathrm{I}_{2}, 16$ (96\%). (ii) Iodosobenzenediacetate, $\mathrm{CF}_{3} \mathrm{SO}_{3} \mathrm{H}, 14$ (46\%).

For the preparation of compound $\mathbf{1 2}$ we first synthesized the novel (2-iodoethynyl)(phenyl)iodonium triflate 14, by using a procedure similar to that used for the preparation of [(2-trimethylsilyl)ethynyl](phenyl)iodonium triflate. ${ }^{8}$ Thus, iodination of (trimethylsilyl)acetylene 15 by reaction with $n$-BuLi and iodine in THF, as described, ${ }^{9}$ gave 1-iodo-2(trimethylsilyl)acetylene 16. Reaction of compound 16 with iodosobenzene diacetate (IBDA) and triflic acid gave the crude iodonium triflate 14, as light brown solid containing some acetic acid (Scheme 3). After crystallization from $\mathrm{MeCN} / \mathrm{CH}_{2} \mathrm{Cl}_{2} 1: 3$, triflate 14 was obtained in $46 \%$ yield, as white solid, quite stable in a dry argon atmosphere at $5{ }^{\circ} \mathrm{C}$. This procedure is more simple than that described for the preparation of (2-chloroethynyl)(phenyl)iodonium triflate, ${ }^{10}$ which implies reaction of (2-chloroethynyl)tributylstannane with (cyano)(phenyl)iodonium triflate, both not commercially available.

Reaction of crude diene 5 with the triflate $\mathbf{1 4}$ in $\mathrm{MeCN}$ at room temperature for $20 \mathrm{~h}$ gave the expected Diels-Alder adduct $\mathbf{9}$ in 79\% yield. Reaction of $\mathbf{9}$ with NaI/CuI in MeCN, following the procedure described by Stang et al. ${ }^{11}$ in related cases, gave diiodide 12 in $46 \%$ yield (Scheme 2). The stereochemistry of both compounds was clearly established as for $\mathbf{6}$ on the basis of the different NMR data, especially the ${ }^{1} \mathrm{H} /{ }^{1} \mathrm{H}$ NOESY experiments, thus showing that the addition of triflate $\mathbf{1 4}$ to the diene $\mathbf{5}$ had taken place, as in the precedent cases, by the less hindered methoxycarbonyl face. Worthy of note, reaction of the iodonium triflate $\mathbf{9}$ with aqueous sodium hydroxide gave a mixture of the volatile iodobenzene (traces), diiodide 12 (35\%) and iodoketone 13 (23\%). These facts suggest competition of the nucleophilic attack of the hydroxide ion to the ipso phenyl and the 2-norbornadiene positions. The enol, initially formed by substitution of the phenyliodonium group by hydroxide, would then tautomerize to the more stable 3-endoiodoketone 13.

1,2-Diiodoethylene derivatives related to 12 have been prepared through Diels-Alder reactions using bis[phenyl[[(trifluoromethyl)sulfonyl]oxy]iodo]acetylene ${ }^{12}$ as the dienophile, followed by reaction of the adducts with NaI/CuI. ${ }^{11}$ However, this dienophile is much less stable and more difficult to prepare than 14. 


\section{Conclusions}

In conclusion we have prepared methyl 1-benzylcyclopenta-2,4-diene-1-carboxylate and studied its Diels-Alder reactions with maleic anhydride, cis-1,2-bis(phenylsulfonyl)ethylene and (2iodoethynyl)(phenyl)iodonium triflate, and further transformations of the obtained adducts. Of special interest is the adduct with the above iodonium triflate, that has been transformed into methyl 1-benzyl-2,3-diiodonorbornadiene-7-carboxylate. Work is in progress to apply the described methodologies for the preparation of more complex polycyclic compounds.

\section{Experimental Section}

General. Melting points were determined with a MFB 595010 M Gallenkamp melting point apparatus. ${ }^{1} \mathrm{H}$ NMR spectra were recorded on Varian Gemini-300 (300 MHz), Varian Mercury400 (400 MHz), or Varian VXR-500 (500 MHz) spectrometers. ${ }^{13} \mathrm{C}$ NMR spectra were recorded on Varian Mercury-400 (100.6 MHz), or Varian VXR-500 (125.8 MHz) spectrometers. The ${ }^{1} \mathrm{H} /{ }^{1} \mathrm{H}$ homocorrelation spectra (COSY and NOESY) and the one bond and long range ${ }^{1} \mathrm{H} /{ }^{13} \mathrm{C}$ heterocorrelation spectra (gHSQC and gHMBC, respectively) were performed on a Varian VXR500 spectrometer. Chemical shifts are given in $\delta$ scale and the coupling constants in Hz. IR spectra were registered on a FTIR Perkin-Elmer Spectrum RX1 spectrometer usually with the attenuated total reflectance (ATR) technique. High resolution MS spectra were performed in a LC/MSD-TOF spectrometer at the Serveis Científico-Tècnics of the University of Barcelona. The elemental analyses were determined in a Carlo Erba model 1106 equipment at the IIQAB (CSIC) of Barcelona, Spain. For the column chromatography, silica gel 60 AC (35-70 $\mu \mathrm{M}$, SDS, ref. 2000027) was used. Thin-layer chromatography (TLC) was performed on aluminum-backed sheets with silica gel $60 \mathrm{~F}_{254}$ (Merck, ref. 1.05554) and spots were visualized with UV light, a $1 \%$ aqueous solution of $\mathrm{KMnO}_{4}$ or by placing the sheets in an iodine atmosphere.

Methyl rac-1-benzyl-2-oxocyclopent-3-enecarboxylate (3). To a cold $\left(0{ }^{\circ} \mathrm{C}\right)$ solution of keto ester 1 (614 mg, $2.64 \mathrm{mmol})$ and anhydrous $\mathrm{Et}_{3} \mathrm{~N}(1.8 \mathrm{~mL}, 1.33 \mathrm{~g}, 13.2 \mathrm{mmol})$ in anhydrous $\mathrm{CH}_{2} \mathrm{Cl}_{2}(4.5 \mathrm{~mL})$, trimethylsilyl trifluoromethanesulfonate $(0.72 \mathrm{~mL}, 880 \mathrm{mg}, 4.0 \mathrm{mmol})$ was added dropwise and the mixture was stirred at room temperature for $30 \mathrm{~min}$. The mixture was cooled to $0{ }^{\circ} \mathrm{C}$ and a saturated solution of $\mathrm{NaHCO}_{3}(3 \mathrm{~mL})$ was added. The organic phase was separated, dried (anhydrous $\mathrm{Na}_{2} \mathrm{SO}_{4}$ ) and concentrated to dryness in vacuo. The residue was taken in hexane $(5 \mathrm{~mL})$ and was washed with water $(3 \mathrm{~mL})$. The dried organic phase (anhydrous $\mathrm{Na}_{2} \mathrm{SO}_{4}$ ) was concentrated in vacuo to give methyl 1-benzyl-2-(trimethylsilyloxy)ciclopent-2enecarboxylate 2 (750 $\mathrm{mg}$ ) as yellow oil, that was used as such in the next step. $R_{f}=0.41$ (silica gel, $10 \mathrm{~cm}$, hexane / EtOAc 7:3). ${ }^{1} \mathrm{H}$ NMR $\left(300 \mathrm{MHz}, \mathrm{CDCl}_{3}\right) \delta 0.23\left(\mathrm{~s}, 9 \mathrm{H}, \mathrm{Si}\left(\mathrm{CH}_{3}\right)_{3}\right), 1.62-$ $1.72(\mathrm{~m}, 1 \mathrm{H})$ and $1.84-1.92(\mathrm{~m}, 1 \mathrm{H})\left(5-\mathrm{H}_{\text {cis }}\right.$ and $\left.5-\mathrm{H}_{\text {trans }}\right), 2.07-2.25$ (complex signal, $2 \mathrm{H}, 4-\mathrm{H}_{c i s}$ 
and 4- $\mathrm{H}_{\text {trans }}$ ), 3.01 (d, $\left.J=13.5 \mathrm{~Hz}, 1 \mathrm{H}\right)$ and 3.12 (d, $\left.J=13.5 \mathrm{~Hz}, 1 \mathrm{H}\right)\left(\mathrm{CH}_{2}-\mathrm{Ph}\right), 3.70$ (s, $3 \mathrm{H}$, $\mathrm{OCH}_{3}$ ), 4.62 (t, $\left.J=2.4 \mathrm{~Hz}, 1 \mathrm{H}, 3-\mathrm{H}\right), 7.18-7.23$ (complex signal, 5H, Ar-H).

To a solution of the above trimethylsilyl ether $2(750 \mathrm{mg})$ in anhydrous DMSO (4.6 $\mathrm{mL})$, $\mathrm{Pd}(\mathrm{OAc})_{2}$ (30 mg, $0.13 \mathrm{mmol}$ ) was added and the red-brown mixture was vigorously stirred for $24 \mathrm{~h}$ while oxygen was being bubbled through the mixture via a syringe. The mixture was diluted with EtOAc $(10 \mathrm{~mL})$ and was washed with water $(8 \mathrm{~mL})$. The aqueous phase was extracted with EtOAc $(2 \times 10 \mathrm{~mL})$ and the combined organic phase and extracts were washed with brine $(2 \times 8$ $\mathrm{mL}$ ), dried (anhydrous $\mathrm{Na}_{2} \mathrm{SO}_{4}$ ) and concentrated in vacuo to give a brown oily residue (605 $\mathrm{mg}$ ). Column chromatography of the above residue (silica gel, 35-70 $\mu \mathrm{m}, 12 \mathrm{~g}$, hexane / EtOAc mixtures) gave in order of elution, starting keto ester 1 (57 mg, hexane / EtOAc 99:1) and enone 3 (496 mg, 82\%, hexane / EtOAc 95:5) as colorless oil, $R_{f}=0.29$ (silica gel, $10 \mathrm{~cm}$, hexane / EtOAc 7:3). The ${ }^{1} \mathrm{H}$ NMR spectrum is concordant with that described. ${ }^{4}$

Methyl 1-benzylcyclopenta-2,4-diene-1-carboxylate (5). To a cold $\left(0{ }^{\circ} \mathrm{C}\right)$ solution of enone 3 (382 mg, $1.66 \mathrm{mmol}$ ) and $\mathrm{CeCl}_{3} \cdot 7 \mathrm{H}_{2} \mathrm{O}$ (803 mg, $\left.2.16 \mathrm{mmol}\right)$ in $\mathrm{MeOH}(15 \mathrm{~mL}), \mathrm{NaBH}_{4}(246$ $\mathrm{mg}, 6.5 \mathrm{mmol}$ ) was added and the solution was stirred at room temperature for $1 \mathrm{~h}$. A saturated aqueous solution of $\mathrm{Na}_{2} \mathrm{SO}_{4}(10 \mathrm{~mL})$ was added and the mixture was extracted with $\mathrm{CH}_{2} \mathrm{Cl}_{2}$ $(3 \times 50 \mathrm{~mL})$. The combined organic phases were dried (anhydrous $\mathrm{Na}_{2} \mathrm{SO}_{4}$ ) and concentrated in vacuo to give hydroxy ester 4 (384 mg, quantitative yield) as colorless oil, $R_{f}=0.18$ (silica gel, 9 $\mathrm{cm}$, hexane / EtOAc 7:3). ${ }^{1} \mathrm{H}$ NMR (300 $\left.\mathrm{MHz} \mathrm{CDCl}_{3}\right) \delta 1.72$ (broad s, 1H, OH), 2.47 (dq, $J=$ $\left.17.3 \mathrm{~Hz}, J^{\prime}=2.0 \mathrm{~Hz}, 1 \mathrm{H}\right)$ and $2.78\left(\mathrm{dd}, J=17.3 \mathrm{~Hz}, J^{\prime}=2.0 \mathrm{~Hz}, 1 \mathrm{H}\right)\left(5-\mathrm{H}_{\text {cis }}\right.$ and 5- $\left.\mathrm{H}_{\text {trans }}\right), 2.88$ (d, $J=13.8 \mathrm{~Hz}, 1 \mathrm{H})$ and $3.32(\mathrm{~d}, J=13.8 \mathrm{~Hz}, 1 \mathrm{H})\left(\mathrm{CH}_{2}-\mathrm{Ph}\right), 3.64$ (s, $\left.3 \mathrm{H}, \mathrm{OCH}_{3}\right), 5.00$ (m, 1H, 2H), 5.82 (dq, $\left.J=5.7 \mathrm{~Hz}, J^{\prime}=2.1 \mathrm{~Hz}, 1 \mathrm{H}, 4-\mathrm{H}\right), 5.95$ (ddt, $J=5.7 \mathrm{~Hz}, J^{\prime}=1.2 \mathrm{~Hz}, J^{\prime}$ ' $=2.4 \mathrm{~Hz}$, 1H, 3-H), 7.10-7.14 (m, 2H) and 7.18-7.29 (complex signal, 3H) (Ar-H).

To a solution of alcohol 4 (639 mg, $2.75 \mathrm{mmol})$ in benzene $(60 \mathrm{~mL}), p-\mathrm{TsOH} \cdot \mathrm{H}_{2} \mathrm{O}(27 \mathrm{mg}, 0.14$ mmol) was added and the solution was heated under reflux for $18 \mathrm{~h}$ with azeotropic elimination of water with a Dean-Stark equipment. Then, the solution was allowed to cool to room temperature and was treated with saturated aqueous solution of $\mathrm{NaHCO}_{3}(12 \mathrm{~mL})$. The organic phase was separated and the aqueous one was extracted with diethyl ether $(1 \times 20 \mathrm{~mL}$ and $2 \times 15$ $\mathrm{mL})$. The combined organic phase and extracts were washed with water $(2 \times 15 \mathrm{~mL})$ and brine ( $2 \times 15 \mathrm{~mL}$ ), dried (anhydrous $\mathrm{Na}_{2} \mathrm{SO}_{4}$ ) and concentrated in vacuo to give crude 5 , as brown oil (587 mg, quantitative yield). Column chromatography of the above oil (silica gel, 35-70 $\mu \mathrm{m}, 12$ g, hexane / EtOAc mixtures) gave, on elution with hexane / EtOAc 99:1, diene 5 (387 mg, 66\%) as a clear brown oil, $R_{f}=0.36$ (silica gel, $10 \mathrm{~cm}$, hexane / AcOEt 9:1). IR (NaCl): $v$ 3061, 3028, 2950, 2925, 2854, 1728 (C=O st), 1602, 1495, 1453, 1434, 1369, 1308, 1257, 1218, 1081, 1041, 792, 775, 763, 732, 716, $701 \mathrm{~cm}^{-1} .{ }^{1} \mathrm{H}$ NMR (400 MHz, $\left.\mathrm{CDCl}_{3}\right): \delta 3.12$ (s, 2H, CH $\left.2-\mathrm{Ph}\right), 3.60$ (s, $\left.3 \mathrm{H}, \mathrm{OCH}_{3}\right), 6.32(\mathrm{~m}, 2 \mathrm{H}, 3(4)-\mathrm{H}), 6.43(\mathrm{~m}, 2 \mathrm{H}, 2(5)-\mathrm{H}), 7.14-7.17(\mathrm{~m}, 2 \mathrm{H})$ and 7.20-7.26 (complex signal, 3H) (Ar-H). ${ }^{13} \mathrm{C}$ NMR (100.6 MHz, $\left.\mathrm{CDCl}_{3}\right): \delta 40.8\left(\mathrm{CH}_{2}, \mathrm{CH}_{2}-\mathrm{Ph}\right), 52.2\left(\mathrm{CH}_{3}\right.$, $\left.\mathrm{OCH}_{3}\right), 67.8$ (C, C1), 126.6 (CH, Ar-C4), 127.8 [CH, Ar-C3(5)], 129.7 [CH, Ar-C2(6)], 131.8 [CH, C3(4)], 137.5 (C, Ar-C1), 138.4 [CH, C2(5)], 172.0 (C, COOMe). HRMS (ESI-TOF): 
calcd. for $\left[\mathrm{C}_{14} \mathrm{H}_{14} \mathrm{O}_{2}+\mathrm{H}\right]^{+}:$215.1066. Found: 215.1069. Anal. calcd. for $\mathrm{C}_{14} \mathrm{H}_{14} \mathrm{O}_{2}$ : C, 78.48; $\mathrm{H}$, 6.59. Found: C, 78.55; H, 6.97.

Dimethyl (1RS,3aRS,4SR,7RS,7aRS,8RS)-1,8-dibenzyl-3a,4,7,7a-tetrahydro-1H-4,7-methanoindene1,8-dicarboxylate (6). A sample of crude diene 5 (106 $\mathrm{mg}, 0.23 \mathrm{mmol}$ ) was kept at room temperature for 4 months. The product thus formed was subjected to column chromatography (silica gel, 35-70 $\mu \mathrm{m}, 10 \mathrm{~g}$, hexane / EtOAc mixtures). On elution with hexane / EtOAc 99:1, diene 5 (14 mg) was isolated and on elution with hexane / EtOAc 98:2, dimer 6 (75 mg) was obtained as light yellow solid. Crystallization from $\mathrm{MeOH}(1.5 \mathrm{~mL})$ gave pure 6 (62 mg, 58\% from 3), as white solid, mp 144-145 ${ }^{\circ} \mathrm{C}, R_{f}=0.31$ (silica gel, $10 \mathrm{~cm}$, hexane / EtOAc 8:2). IR (ATR): v 3081, 3025, 3001, 2951, 2929, 2850, 1723 (C=O st), 1492, 1441, 1324, 1303, 1266, 1227, 1189, 1104, 1088, 1079, 1057, 1041, 1024, 951, 781, 769, 754, 741, 723, 714, 697, 602 $\mathrm{cm}^{-1} .{ }^{1} \mathrm{H}$ NMR (500 MHz, $\mathrm{CDCl}_{3}$ ): $\delta 2.67$ (d, $J=13.0 \mathrm{~Hz}, 1 \mathrm{H}, 1-\mathrm{CH}_{\mathrm{a}}-\mathrm{Ph}$ ), 2.81 (dd, $J=8.0 \mathrm{~Hz}$, $\left.J^{\prime}=3.5 \mathrm{~Hz}, 1 \mathrm{H}, 7 \mathrm{a}-\mathrm{H}\right), 2.90$ (d, $\left.J=14.0 \mathrm{~Hz}, 1 \mathrm{H}, 8-\mathrm{CH}_{\mathrm{a}}-\mathrm{Ph}\right), 2.95$ (m, 1H, 4-H), 2.98 (d, $J=14.0$ $\mathrm{Hz}, 1 \mathrm{H}, 8-\mathrm{CH}_{\mathrm{b}}-\mathrm{Ph}$ ), 3.14 (d, $\left.J=13.0 \mathrm{~Hz}, 1 \mathrm{H}, 1-\mathrm{CH}_{\mathrm{b}}-\mathrm{Ph}\right), 3.30$ (m, 1H, 7-H), 3.34 (m, 1H, 3a-H), 3.60 (s, 3H, 8-COOCH ), 3.62 (s, 3H, 1-COOCH $\mathrm{CO}_{3}, 5.43$ (dd, $J=6.0 \mathrm{~Hz}, J^{\prime}=2.0 \mathrm{~Hz}, 1 \mathrm{H}, 3-\mathrm{H}$ ), 5.56 (dd, $\left.J=6.0 \mathrm{~Hz}, J^{\prime}=2.0 \mathrm{~Hz}, 1 \mathrm{H}, 2-\mathrm{H}\right), 5.72$ (ddd, $J=6.0 \mathrm{~Hz}, J^{\prime}=3.0 \mathrm{~Hz}, J^{\prime}$ ' = $1.5 \mathrm{~Hz}, 1 \mathrm{H}$, 6-H), 5.89 (dd, $\left.J^{\prime}=6.0 \mathrm{~Hz}, J^{\prime \prime}=3.0 \mathrm{~Hz}, 1 \mathrm{H}, 5-\mathrm{H}\right), 6.94$ [dm, $J=8.5 \mathrm{~Hz}, 2 \mathrm{H}, 8-\mathrm{CH}_{2}-\mathrm{Ar}-2(6)-H$ ], 6.98 [ddm, $\left.J=8.0 \mathrm{~Hz}, J^{\prime}=2.0 \mathrm{~Hz}, 2 \mathrm{H}, 1-\mathrm{CH}_{2}-\mathrm{Ar}-2(6)-H\right]$, 7.15-7.24 [m, 6H, 1-CH $-\mathrm{Ar}-3(5)-H$, 8-CH $2-\mathrm{Ar}-3(5)-H, 1-\mathrm{CH}_{2}-\mathrm{Ar}-4-\mathrm{H}$ and 8- $\left.\mathrm{CH}_{2}-\mathrm{Ar}-4-H\right] .{ }^{13} \mathrm{C} \mathrm{NMR}\left(125.8 \mathrm{MHz}, \mathrm{CDCl}_{3}\right): \delta 37.5$ $\left(\mathrm{CH}_{2}, 8-\mathrm{CH}_{2}-\mathrm{Ph}\right), 49.6\left(\mathrm{CH}_{2}, 1-\mathrm{CH}_{2}-\mathrm{Ph}\right), 50.3(\mathrm{CH}, \mathrm{C} 4), 50.7(\mathrm{CH}, \mathrm{C7a}), 51.2\left(\mathrm{CH}_{3}, 8-\right.$ $\left.\mathrm{COOCH}_{3}\right), 51.4\left(\mathrm{CH}_{3}, 1-\mathrm{COOCH}_{3}\right), 51.5(\mathrm{CH}, \mathrm{C} 3 \mathrm{a}), 51.9(\mathrm{CH}, \mathrm{C} 7), 61.9(\mathrm{C}, \mathrm{C} 1), 75.6(\mathrm{C}, \mathrm{C} 8)$, $126.3\left(\mathrm{CH}, 8-\mathrm{CH}_{2}-\mathrm{Ar}-\mathrm{C4}\right), 126.6$ (CH, 1- $\left.\mathrm{CH}_{2}-\mathrm{Ar}-\mathrm{C4}\right), 127.9$ [CH, 1-CH $\left.-\mathrm{Ar}-\mathrm{C3}(5)\right], 128.0$ [CH, 8-CH $2-\mathrm{Ar}-\mathrm{C3}(5)$ ], 129.1 [CH, 8-CH $2-\mathrm{Ar}-\mathrm{C2}(6)$ ], 129.8 [CH, 1- $\left.\mathrm{CH}_{2}-\mathrm{Ar}-\mathrm{C2}(6)\right], 129.9$ (CH, C6), 132.1 (CH, C3), 134.5 (CH, C5), 135.0 (CH, C2), 136.9 (C, 1- $\left.\mathrm{CH}_{2}-\mathrm{Ar}-\mathrm{C} 1\right), 138.6$ (C, 8- $\mathrm{CH}_{2}-\mathrm{Ar}-$ C1), 174.7 (C, 8-COOCH 3$), 175.5$ (C, 1-COOCH$)_{3}$. HRMS (ESI-TOF): calcd. for $\left[\mathrm{C}_{28} \mathrm{H}_{28} \mathrm{O}_{4}+\mathrm{H}\right]^{+}:$429.2060. Found: 429.2059. Calcd. for $\left[\mathrm{C}_{28} \mathrm{H}_{28} \mathrm{O}_{4}+\mathrm{NH}_{4}\right]^{+}:$446.2326. Found: 446.2320. Anal. calcd. for $\mathrm{C}_{28} \mathrm{H}_{28} \mathrm{O}_{4}$ : C, 78.48; H, 6.59. Found: C, 78.31; H, 6.55.

(1R,2S,3R,4S,7r)-7-Benzyl-7-methoxycarbonylbicyclo[2.2.1]hept-5-ene-2,3-dicarboxylic

anhydride (7). A solution of crude diene 5 (221 mg, $1.03 \mathrm{mmol}$ ) and maleic anhydride (152 mg, $1.55 \mathrm{mmol})$ in anhydrous toluene (4 mL) was heated under reflux for $4 \mathrm{~h}$. The solution was allowed to cool to room temperature and concentrated in vacuo to give a brown viscous oil that was subjected to column chromatography (silica gel, 35-70 $\mu \mathrm{m}, 12 \mathrm{~g}$, hexane / EtOAc mixtures. On elution with hexane / EtOAc 95:5, anhydride 7 (289 mg, 90\%, from enone 3) was obtained as brown solid. Crystallization of the above product from toluene / pentane 5:6 $(1.1 \mathrm{~mL})$, provided the analytical sample of 7 as white solid (146 mg, 45\%), mp $145-146{ }^{\circ} \mathrm{C}$. $R_{f}=0.87$ (silica gel, 10 cm, $\mathrm{CH}_{2} \mathrm{Cl}_{2} / \mathrm{MeOH}$ 8:2). IR (KBr): v 3448, 3018, 2963, 2938, 1855, 1776 and 1739 (C=O st), 1442, 1328, 1295, 1260, 1237, 1225, 1204, 1129, 1090, 1063, 1044, 930, 912, 759, 700, 670, 625 $\mathrm{cm}^{-1} .{ }^{1} \mathrm{H}$ NMR (300 MHz, $\mathrm{CDCl}_{3}$ ): $\delta 2.98$ (s, 2H, $\mathrm{CH}_{2}-\mathrm{Ph}$ ), 3.55 [m, 2H, 1(4)-H], 3.62 (s, 3H, $\mathrm{OCH}_{3}$ ), 3.66 (dd, $\left.J=3.0 \mathrm{~Hz}, J^{\prime}=1.5 \mathrm{~Hz}, 2 \mathrm{H}, 2(3)-\mathrm{H}\right), 6.41$ [t, $\left.J=2.0 \mathrm{~Hz}, 2 \mathrm{H}, 5(6)-\mathrm{H}\right], 6.92$ (ddm, $J=7.6 \mathrm{~Hz}, J^{\prime}=2.0 \mathrm{~Hz}, 2 \mathrm{H}$, Ar- $\mathrm{H}_{\text {ortho }}$ ), 7.22-7.29 (complex signal, 3H, Ar- $\mathrm{H}_{\text {meta }}$ and Ar- 
$\left.\mathrm{H}_{\text {para }}\right) .{ }^{13} \mathrm{C}$ NMR (100.6 MHz, $\left.\mathrm{CDCl}_{3}\right): \delta 36.2\left(\mathrm{CH}_{2}, \mathrm{CH}_{2}-\mathrm{Ph}\right), 45.8[\mathrm{CH}, \mathrm{C} 2(3)], 50.3[\mathrm{CH}$, C1(4)], $52.0\left(\mathrm{CH}_{3}, \mathrm{OCH}_{3}\right), 77.8$ (C, C7), 127.1 (CH, Ar-C4), 128.4 [CH, Ar-C3(5)], 128.8 [CH, Ar-C2(6)], 133.9 [CH, C5(6)], 136.8 (C, Ar-C1), 170.4 [C, 2(3)-COO], 172.7 (C, COOMe). HRMS (ESI-TOF): calcd. for $\left[\mathrm{C}_{18} \mathrm{H}_{16} \mathrm{O}_{5}+\mathrm{H}\right]^{+}$: 313.1071. Found: 313.1077. Calcd. for $\left[\mathrm{C}_{18} \mathrm{H}_{16} \mathrm{O}_{5}+\mathrm{Na}\right]^{+}$: 335.0890. Found: 335.0891. Anal. calcd. for $\mathrm{C}_{18} \mathrm{H}_{16} \mathrm{O}_{5}$ : C, 69.22; $\mathrm{H}, 5.16$. Found: C, 68.88; H, 5.24.

Methyl (1R,4S,5S,6R,7s)-7-benzyl-5,6-bis(phenylsulfonyl)bicyclo[2.2.1]hept-2-ene-7-carboxylate (8). A solution of crude diene 5 (296 $\mathrm{mg}, 1.38 \mathrm{mmol})$ and cis-1,2bis(phenylsulfonyl)ethylene (468 mg, $1.52 \mathrm{mmols}$ ) in anhydrous toluene (5 mL) was heated to $100{ }^{\circ} \mathrm{C}$ for $15 \mathrm{~h}$. The solution was allowed to cool to room temperature and concentrated in vacuo to give a brown viscous oil that was subjected to column chromatography (silica gel, 35$70 \mu \mathrm{m}, 50 \mathrm{~g}$, hexane / EtOAc mixtures). In order of elution, dimer 6 (85 mg), starting disulfone (140 mg) and adduct 8 as light brown solid (438 mg, 61\% from enone 3), were obtained. The analytical sample of adduct 8 (232 $\mathrm{mg}$ ) was obtained, as white solid, by crystallization of a sample of the above product (340 mg) from $\mathrm{CH}_{2} \mathrm{Cl}_{2} / \mathrm{MeOH} 4: 5(0.9 \mathrm{~mL}), \mathrm{mp} 236-237^{\circ} \mathrm{C} . R_{f}=$ 0.15 (silica gel, $10 \mathrm{~cm}$, hexane / EtOAc 6:4). IR (ATR): v 3001, 2945, 1726 (C=O st), 1584,

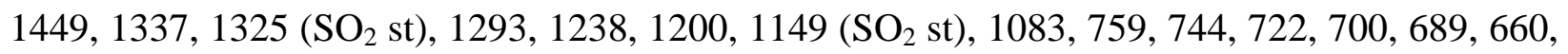
611, 601, 590, $578 \mathrm{~cm}^{-1} .{ }^{1} \mathrm{H}$ NMR (300 MHz, $\mathrm{CDCl}_{3}$ ): $\delta 2.88$ (s, 2H, $\mathrm{CH}_{2}-\mathrm{Ph}$ ), 3.25 [broad s, 2H, 1(4)-H], 3.46 (s, 3H, $\mathrm{OCH}_{3}$ ), 4.15 (t, $\left.J=1.4 \mathrm{~Hz}, 2 \mathrm{H}, 5(6)-\mathrm{H}\right), 6.71$ [t, $J=2.0 \mathrm{~Hz}, 2 \mathrm{H}, 2(3)-\mathrm{H}$ ], 6.81-6.84 (dm, $J=7.5 \mathrm{~Hz}, 2 \mathrm{H}$, Ar- $\mathrm{H}_{\text {ortho }}$ benzyl), 7.16-7.21 (complex signal, 3H, Ar-H $\mathrm{H}_{\text {meta }}$ and Ar- $\mathrm{H}_{\text {para }}$ benzyl), 7.55 (tm, $J=7.5 \mathrm{~Hz}, 4 \mathrm{H}$, Ar- $\mathrm{H}_{\text {meta }}-\mathrm{SO}_{2} \mathrm{Ph}$ ), 7.63-7.67 (tm, $J=7.5 \mathrm{~Hz}, 2 \mathrm{H}$, Ar$\mathrm{H}_{\text {para }}-\mathrm{SO}_{2} \mathrm{Ph}$ ), 7.96-7.98 (dm, $\left.J=7.5 \mathrm{~Hz}, 4 \mathrm{H}, \mathrm{Ar}-\mathrm{H}_{\text {ortho }}-\mathrm{SO}_{2} \mathrm{Ph}\right) .{ }^{13} \mathrm{C}$ NMR $\left(100.6 \mathrm{MHz}, \mathrm{CDCl}_{3}\right)$ : $\delta 35.2\left(\mathrm{CH}_{2}, \mathrm{CH}_{2}-\mathrm{Ph}\right), 51.9\left(\mathrm{CH}_{3}, \mathrm{OCH}_{3}\right), 52.3$ [CH, C1(4)], 70.0 [CH, C5(6)], 71.5 (C, C7), 127.0 (CH, Ar-C4 benzyl), 128.3 [CH, Ar-C3(5) benzyl], 128.5 [CH, Ar-C2(6), SO $\mathrm{Ph}_{2} 128.8$ [CH, Ar-C2(6) benzyl], 129.0 (CH, Ar-C4 -SO $2 \mathrm{Ph}$ ), 132.9 [CH, C2(3)], 133.7 (CH, Ar-C3(5) $\mathrm{SO}_{2} \mathrm{Ph}$ ), 136.5 (C, Ar-C1 benzyl), 140.9 (C, Ar-C1 -SO $2 \mathrm{Ph}$ ), 172.6 (C, COOMe). HRMS (ESITOF): calcd. for $\left[\mathrm{C}_{28} \mathrm{H}_{26} \mathrm{O}_{6} \mathrm{~S}_{2}+\mathrm{H}\right]^{+}$: 523.1244. Found: 523.1238. Calcd. for $\left[\mathrm{C}_{28} \mathrm{H}_{26} \mathrm{O}_{6} \mathrm{~S}_{2}+\mathrm{NH}_{4}\right]^{+}$: 540.1509. Found: 540.1500. Anal. calcd. for $\mathrm{C}_{28} \mathrm{H}_{26} \mathrm{O}_{6} \mathrm{~S}_{2}$ : C, 64.35; H, 5.01. Found: C, 64.09; H, 4.85.

Methyl 7-benzylbicyclo[2.2.1]hepta-2.5-diene-7-carboxylate (10). To a well stirred suspension of compound 8 (735 mg, $1.41 \mathrm{mmol}$ ) in anhydrous 1,4-dioxane (15 mL) under an Ar atmosphere, $2 \%$ sodium amalgam $(12.9 \mathrm{~g}, 11.3 \mathrm{mmol}$ ) was added portionwise within $1 \mathrm{~h}$ and then, the mixture was vigorously stirred for $20 \mathrm{~h}$ at room temperature. The organic phase was separated and the residue was washed with EtOAc $(2 \times 4 \mathrm{~mL})$. The combined organic phase and washings were concentrated in vacuo and the obtained brown oily residue was subjected to column chromatography (silica gel, 35-70 $\mu \mathrm{m}, 25$ g, hexane / EtOAc mixtures). On elution with hexane / EtOAc 99:1, compound 10 (178 mg, 53\%) was isolated as yellow oil. $R_{f}=0.66$ (silica gel, 10 cm, hexane / EtOAc 8:2). IR (ATR): v 3071, 3040, 3000, 2948, 1726 (C=O st), 1496, 1457, 1433, 1318, 1277, 1228, 1200, 1171, 1099, 1083, 1035, 733, 700, 656, 602, $576 \mathrm{~cm}^{-1} .{ }^{1} \mathrm{H}$ NMR (500 MHz, $\left.\mathrm{CDCl}_{3}\right): \delta 3.02\left(\mathrm{~s}, 2 \mathrm{H}, \mathrm{CH}_{2}-\mathrm{Ph}\right), 3.43$ (s, $\left.3 \mathrm{H}, \mathrm{OCH}_{3}\right), 3.64$ [quint, $J=2.0 \mathrm{~Hz}, 2 \mathrm{H}$, 
1(4)-H], 6.73 [t, $J=2.0 \mathrm{~Hz}, 2 \mathrm{H}, 2(3)-\mathrm{H}], 6.77$ [t, $J=2.0 \mathrm{~Hz}, 2 \mathrm{H}, 5(6)-\mathrm{H}], 6.98$ (dm, $J=8.5 \mathrm{~Hz}$, 2H, Ar- $\mathrm{H}_{\text {ortho }}$ ), 7.19 (tm, $\left.J=7.5 \mathrm{~Hz}, 1 \mathrm{H}, \mathrm{Ar}-\mathrm{H}_{\text {para }}\right), 7.25$ (m, 2H, Ar- $\left.\mathrm{H}_{\text {meta }}\right) .{ }^{13} \mathrm{C}$ NMR (100.6 $\left.\mathrm{MHz}, \mathrm{CDCl}_{3}\right): \delta 39.0\left(\mathrm{CH}_{2}, \mathrm{CH}_{2}-\mathrm{Ph}\right), 51.0\left(\mathrm{CH}_{3}, \mathrm{OCH}_{3}\right), 55.0$ [CH, C1(4)], 96.1 (C, C7), 126.3

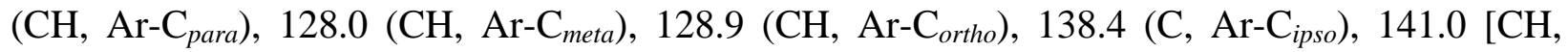
C5(6)], 143.1 [CH, C2(3)], 174.9 (C, COO). HRMS (ESI-TOF): calcd. for $\left[\mathrm{C}_{16} \mathrm{H}_{16} \mathrm{O}_{2}+\mathrm{H}\right]^{+}$: 241.1223. Found: 241.1231. Calcd. for $\left[\mathrm{C}_{16} \mathrm{H}_{16} \mathrm{O}_{2}+\mathrm{NH}_{4}\right]^{+}$: 258.1489. Found: 258.1492. Anal. calcd. for $\mathrm{C}_{16} \mathrm{H}_{16} \mathrm{O}_{2}$ : C, 79.97; H, 6.71. Found: C, 79.91; H, 6,82.

Reduction of disulfone (9) with $2 \% \mathrm{Na}(\mathrm{Hg})$ in methanol: formation of methyl (1RS,2rs,3SR,6SR)-3-benzyltricyclo[2.2.1.0 $\left.{ }^{2,6}\right]$ heptane-3-carboxylate (11) and diene (10). To a well stirred suspension of compound 8 (190 mg, $0.36 \mathrm{mmol})$ and $\mathrm{NaH}_{2} \mathrm{PO}_{4} \cdot \mathrm{H}_{2} \mathrm{O}(727 \mathrm{mg}, 5.27$ $\mathrm{mmol}$ ) in $\mathrm{MeOH}(15 \mathrm{~mL}$ ) under an Ar atmosphere, 2\% sodium amalgam (3.34 g, $2.9 \mathrm{mmol}$ ) was added portionwise within $1 \mathrm{~h}$ and then, the mixture was vigorously stirred for $20 \mathrm{~h}$ at room temperature. The organic phase was separated and the residue was washed with $\mathrm{MeOH}(2 \times 2$ $\mathrm{mL}$ ). The combined organic phase and washings were concentrated in vacuo and the gray solid residue was subjected to column chromatography (silica gel, 35-70 $\mu \mathrm{m}, 10 \mathrm{~g}$, hexane / EtOAc mixtures). In order of elution, slightly impure compound 11 (21 mg, about 25\%) and a mixture of diene 10 and compound 11 (31 mg) were isolated. NMR data of compound 11: ${ }^{1} \mathrm{H}$ NMR (500 $\mathrm{MHz}_{\mathrm{CDCl}}$ ): $\delta$ 1.21-1.39 (complex signal, 6H, 1-H, 2-H, 5- $\mathrm{H}_{\text {endo }}, 6-\mathrm{H}, 7-\mathrm{H}_{\text {exo }}, 7-\mathrm{H}_{\text {endo }}$ ), 1.89 (dm, $\left.J=11.2 \mathrm{~Hz}, 1 \mathrm{H}, 5-\mathrm{H}_{e x o}\right), 2.06$ (broad s, 1H, 4-H), 2.68 (d, $\left.J=13.2 \mathrm{~Hz}, 1 \mathrm{H}\right)$ and 2.91 (d, $J=$ $13.2 \mathrm{~Hz}, 1 \mathrm{H})\left(\mathrm{CH}_{2}-\mathrm{Ph}\right), 3.51$ (s, 3H, $\left.\mathrm{OCH}_{3}\right), 7.10$ (dm, $\left.J=8.0 \mathrm{~Hz}, 2 \mathrm{H}, \mathrm{Ar}-\mathrm{H}_{\text {ortho }}\right), 7.19$ (tm, $J=$ $\left.7.0 \mathrm{~Hz}, 1 \mathrm{H}, \mathrm{Ar}-\mathrm{H}_{\text {para }}\right), 7.25$ (m, 2H, Ar- $\left.\left.\mathrm{H}_{\text {meta }}\right) .{ }^{13} \mathrm{C} \mathrm{NMR} \mathrm{(100.6} \mathrm{MHz,} \mathrm{CDCl}_{3}\right): \delta 11.3(\mathrm{CH})$ and $11.8(\mathrm{CH})$ (C1 and C6), 16.9 (CH, C2), $31.2\left(\mathrm{CH}_{2}, \mathrm{C} 5\right), 32.7\left(\mathrm{CH}_{2}, \mathrm{C} 7\right), 37.0$ (CH, C4), 39.0 $\left(\mathrm{CH}_{2}, \mathrm{CH}_{2}-\mathrm{Ph}\right), 51.1\left(\mathrm{CH}_{3}, \mathrm{OCH}_{3}\right), 60.9$ (C, C3), $126.3\left(\mathrm{CH}, \mathrm{Ar}-\mathrm{C}_{\text {para }}\right), 128.1$ (CH, Ar-C $\left.{ }_{\text {meta }}\right)$, 129.3 (CH, Ar-C ortho), 138.5 (C, Ar- $\left.\mathrm{C}_{i p s o}\right), 175.8$ (C, COO). HRMS (ESI-TOF): calcd. for $\left[\mathrm{C}_{16} \mathrm{H}_{18} \mathrm{O}_{2}+\mathrm{H}\right]^{+}:$243.1380. Found: 243.1378 .

(1RS,4SR,7SR)-[7-Benzyl-3-iodo-7-(methoxycarbonyl)bicyclo[2.2.1]hepta-2,5-dien-2-yl](phenyl)iodonium triflate (9). To a solution of crude diene 5 (272 $\mathrm{mg}, 1.27 \mathrm{mmol}$ ) in anhydrous MeCN (1 mL), 2-(iodoethynyl)phenyliodonium triflate 14 (426 mg, $0.85 \mathrm{mmol}$ ) was added portionwise over $30 \mathrm{~min}$ and the mixture was vigorously stirred at room temperature for $20 \mathrm{~h}$. More crude diene 5 (100 mg, $0.47 \mathrm{mmol}$ ) was added and the mixture was stirred for $2 \mathrm{~d}$. The solvent was eliminated in vacuo and the solid residue $\left(816 \mathrm{mg}\right.$ ) was crystallized from $\mathrm{CH}_{2} \mathrm{Cl}_{2}$ / $\mathrm{Et}_{2} \mathrm{O}(1: 5)$ (4.8 $\left.\mathrm{mL}\right)$ to give adduct 9 (478 mg, 79\%) as light brown solid, $\mathrm{mp} 161-162{ }^{\circ} \mathrm{C}$. IR (ATR): v 3084, 2944, 1726 (C=O st), 1604, 1567, 1537, 1443, 1315, 1285, 1232, 1200, 1163, 1146, 1099, 1087, 1025, 990, 757, 735, 706, 633, $597 \mathrm{~cm}^{-1} .{ }^{1} \mathrm{H}$ NMR (500 MHz, $\left.\mathrm{CDCl}_{3}\right): \delta 2.94$ (d, $J=14.0 \mathrm{~Hz}, 1 \mathrm{H})$ and $3.03(\mathrm{~d}, J=14.0 \mathrm{~Hz}, 1 \mathrm{H})\left(\mathrm{CH}_{2}-\mathrm{Ph}\right), 3.19\left(\mathrm{~s}, 3 \mathrm{H}, \mathrm{OCH}_{3}\right), 3.90(\mathrm{dt}, J=$ $\left.1.0 \mathrm{~Hz}, J^{\prime}=2.5 \mathrm{~Hz}, 1 \mathrm{H}, 4-\mathrm{H}\right), 4.03$ (dt, $\left.J=1.0 \mathrm{~Hz}, J^{\prime}=2.5 \mathrm{~Hz}, 1 \mathrm{H}, 1-\mathrm{H}\right), 6.81$ (ddd, $J=5.5 \mathrm{~Hz}$,

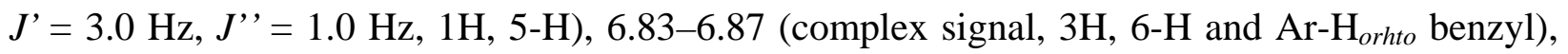
7.19-7.27 (complex signal, 3H, Ar- $\mathrm{H}_{\text {para }}$ and $\mathrm{Ar}-\mathrm{H}_{\text {meta }}$ benzyl), 7.48 (m, 2H, Ar- $\mathrm{H}_{\text {meta }}$ phenyliodonium), 7.65 (tm, $J=7.5 \mathrm{~Hz}, 1 \mathrm{H}, \mathrm{Ar}-\mathrm{H}_{\text {para }}$ phenyliodonium), 7.98 (dm, $J=7.0 \mathrm{~Hz}, 2 \mathrm{H}$, Ar-H $\mathrm{H}_{\text {ortho }}$ phenyliodonium). ${ }^{13} \mathrm{C} \mathrm{NMR}\left(125.8 \mathrm{MHz}, \mathrm{CDCl}_{3}\right): \delta 38.5\left(\mathrm{CH}_{2}, \mathrm{CH}_{2}-\mathrm{Ph}\right), 51.7\left(\mathrm{CH}_{3}\right.$, 
$\mathrm{OCH}_{3}$ ), 64.8 (CH, C1), 67.9 (CH, C4), 96.3 (C, C7), 113.0 (C, Ar-C1 phenyliodonium), 120.1 $\left(\mathrm{CF}_{3}, \mathrm{q}, J=320 \mathrm{~Hz}, \mathrm{CF}_{3} \mathrm{SO}_{3}{ }^{-}\right.$), 127.2 (CH, Ar-C4 benzyl), 128.4 (C, C3), 128.5 [CH, Ar-C3(5) benzyl], 128.8 [CH, Ar-C2(6)], 130.3 (C, C2), 132.0 [CH, Ar-C3(5) phenyliodonium], 132.8 (CH, Ar-C4 phenyliodonium), 135.9 (C, Ar-C1 benzyl), 136.3 [CH, Ar-C2(6) phenyliodonium], 137.0 (CH, C5), 140.5 (CH, C6), 172.1 (C, COOMe). HRMS (ESI-TOF): calcd. for $\left[\mathrm{C}_{23} \mathrm{H}_{19} \mathrm{~F}_{3} \mathrm{I}_{2} \mathrm{O}_{5} \mathrm{~S}-\mathrm{CF}_{3} \mathrm{SO}_{3}\right]^{+}:$568.9469. Found: 568.9463. Anal. calcd. for $\mathrm{C}_{23} \mathrm{H}_{19} \mathrm{~F}_{3} \mathrm{I}_{2} \mathrm{O}_{5} \mathrm{~S}$ : C, 38.46; H, 2.67; I, 35.34; F, 7.94. Found: C, 38.93; H, 2.73; I, 35.86; F, 8.30.

Methyl (1R,4S,7s)-7-benzyl-2,3-diiodobicyclo[2.2.1]hepta-2,5-diene-7-carboxylate (12). To a cold ( -35 to $-40{ }^{\circ} \mathrm{C}$ ) and vigorously stirred suspensión of NaI (254 mg, $\left.1.63 \mathrm{mmol}\right)$ and CuI (311 mg, 1.63 mmols) in anhydrous MeCN (20 mL), triflate $9(1.17 \mathrm{~g}, 1.63 \mathrm{mmol})$ was added. The mixture was allowed to heat to room temperature and was stirred overnight at this temperature. The mixture was filtered and the filtrate was concentrated in vacuo to give a solid residue (1.98 g) that was subjected to column chromatography (silica gel, 35-70 $\mu \mathrm{m}, 80 \mathrm{~g}$, hexane / EtOAc mixtures) to give diiodide 12 as light yellow solid (372 mg, 46\%) on elution with hexane / EtOAc 98:2. An analytical sample of 12 (98 mg) was obtained as light yellow solid by crystallization of a part of the above product (130 mg) from $\mathrm{MeOH}(0.6 \mathrm{~mL}), \mathrm{mp}$ 105.5-106.5 ${ }^{\circ} \mathrm{C}$. IR (ATR): v 3031, 3001, 2946, 1718 (C=O st), 1493, 1453, 1439, 1426, 1316, 1275, 1232, 1201, 1097, 1086, 1033, 1020, 731, 703, 623, $590 \mathrm{~cm}^{-1} .{ }^{1} \mathrm{H}$ NMR (500 MHz, $\left.\mathrm{CDCl}_{3}\right): \delta 2.98$ (s, $2 \mathrm{H}, \mathrm{CH}_{2}-\mathrm{Ph}$ ), 3.51 (s, 3H, $\mathrm{OCH}_{3}$ ), 3.76 [t, $\left.J=2.0 \mathrm{~Hz}, 2 \mathrm{H}, 1(4)-\mathrm{H}\right], 6.85$ [t, $J=2.0 \mathrm{~Hz}, 2 \mathrm{H}, 5(6)-$ $\mathrm{H}$ ], 6.93 (m, 2H, Ar- $\mathrm{H}_{\text {ortho }}$ ), 7.19-7.26 (complex signal, 3H, Ar- $\mathrm{H}_{\text {para }}$ and $\mathrm{Ar}-\mathrm{H}_{\text {meta }}$ ). ${ }^{13} \mathrm{C}$ NMR (125.8 MHz, $\left.\mathrm{CDCl}_{3}\right): \delta 38.7\left(\mathrm{CH}_{2}, \mathrm{CH}_{2}-\mathrm{Ph}\right), 51.5\left(\mathrm{CH}_{3}, \mathrm{OCH}_{3}\right), 66.7$ [CH, C1(4)], 94.9 (C, C7), 114.6 [CH, C2(3)], 126.8 (CH, Ar-C4), 128.3 [CH, Ar-C3(5)], 128.9 [CH, Ar-C2(6)], 137.0 (C, Ar-C1), 138.8 [CH, C5(6)], 172.9 (C, COOMe). HRMS (ESI-TOF): calcd. for $\left[\mathrm{C}_{16} \mathrm{H}_{14} \mathrm{I}_{2} \mathrm{O}_{2}+\mathrm{H}\right]^{+}$: 492.9156. Found: 492.9153 Anal. calcd. for $\mathrm{C}_{16} \mathrm{H}_{14} \mathrm{I}_{2} \mathrm{O}_{2}$ : C, 39.05; H, 2.87; I, 51.58. Found: C, 39.48; H, 2.86; I, 51.84.

Reaction of triflate (9) with aqueous $\mathrm{NaOH}$ : isolation of diiodide (12) and methyl (1RS,4SR,5SR,7RS)-7-benzyl-5-iodo-6-oxobicyclo[2.2.1]hept-5-ene-7-carboxylate (13). Tо а solution of triflate 9 (710 $\mathrm{mg}, 0.99 \mathrm{mmol})$ in $\mathrm{CH}_{2} \mathrm{Cl}_{2}(10 \mathrm{~mL})$, aqueous $1 \mathrm{~N} \mathrm{NaOH}(4 \mathrm{~mL})$ was added and the mixture was vigorously stirred at room temperature for $18 \mathrm{~h}$. The organic phase was separated and the aqueous one was extracted with $\mathrm{CH}_{2} \mathrm{Cl}_{2}(3 \times 3 \mathrm{~mL})$. The combined organic phase and extracts were dried (anhydrous $\mathrm{Na}_{2} \mathrm{SO}_{4}$ ) and concentrated in vacuo to give a brown oily residue (509 mg) that was subjected to column chromatography (silica gel, 35-70 $\mu \mathrm{m}, 25 \mathrm{~g}$, hexane / EtOAc mixtures). In order of elution, iodobenzene (12 mg, hexane), diiodide 12 (170 mg, 35\%, hexane / EtOAc 99:1) and impure iodo ketone 13 (127 mg, hexane / EtOAc 95:5) were isolated. The above iodo ketone $\mathbf{1 3}$ was subjected to a new column chromatography (silica gel, 35-70 $\mu \mathrm{m}, 25 \mathrm{~g}$, pentane / EtOAc mixtures) to give pure product 13 (86 mg, 23\%, pentane / EtOAc 97:3) as light yellow solid, mp 101-102 ${ }^{\circ} \mathrm{C}$. IR (ATR): $v$ 2948, 2922, 2852, 1754, 1728 (C=O st), 1454, 1315, 1238, 1196, 1085, 1035, 909, 736, $701 \mathrm{~cm}^{-1} .{ }^{1} \mathrm{H}$ NMR (500 MHz, $\left.\mathrm{CDCl}_{3}\right)$ : $\delta 3.14(\mathrm{~d}, J=13.5 \mathrm{~Hz}, 1 \mathrm{H})$ and 3.31 (d, $J=13.5 \mathrm{~Hz}, 1 \mathrm{H})\left(\mathrm{CH}_{2}-\mathrm{Ph}\right), 3.35$ (dd, $J=3.0 \mathrm{~Hz}, J$ ' = 1.5 Hz, 1H, 1-H), 3.51 (m, 1H, 4-H), 3.59 (s, 3H, $\mathrm{OCH}_{3}$ ), 4.45 (d, J = 3.0 Hz, 1H, 5- $\mathrm{H}_{\text {exo }}$ ), 6.26 (m, 
1H, 2-H), 6.62 (dd, $\left.J=6.0 \mathrm{~Hz}, J^{\prime}=3.0 \mathrm{~Hz}, 1 \mathrm{H}, 3-\mathrm{H}\right), 6.96$ (m, 2H, Ar- $\mathrm{H}_{\text {ortho }} \mathrm{Ph}$ ), 7.20-7.28 (complex signal, 3H, Ar- $\mathrm{H}_{\text {para }}$ and $\left.\mathrm{Ar}-\mathrm{H}_{\text {meta }}\right) .{ }^{13} \mathrm{C} \mathrm{NMR}\left(125.8 \mathrm{MHz}, \mathrm{CDCl}_{3}\right)$ : $\delta 18.8(\mathrm{CH}, \mathrm{C} 5)$, $36.8\left(\mathrm{CH}_{2}, \mathrm{CH}_{2}-\mathrm{Ph}\right), 52.3\left(\mathrm{CH}_{3}, \mathrm{OCH}_{3}\right), 52.8(\mathrm{CH}, \mathrm{C} 4), 58.1(\mathrm{CH}, \mathrm{C} 1), 73.5(\mathrm{C}, \mathrm{C} 7), 127.1(\mathrm{CH}$, Ar-C4), 128.5 [CH, Ar-C3(5)], 128.9 [CH, Ar-C2(6)], 129.1 (CH, C2), 136.5 (C, Ar-C1), 144.0 (CH, C3), 173.2 (C, COOMe), 204.8 (C, C6). HRMS (ESI-TOF): calcd. for $\left[\mathrm{C}_{16} \mathrm{H}_{15} \mathrm{IO}_{3}+\mathrm{H}\right]^{+}$: 383.0139. Found: 383.0130. Calcd. for $\left[\mathrm{C}_{16} \mathrm{H}_{15} \mathrm{IO}_{3}+\mathrm{NH}_{4}\right]^{+}$: 400.0404. Found: 400.0401. Anal. calcd. for $\mathrm{C}_{16} \mathrm{H}_{15} \mathrm{IO}_{3}$ : C, 50.28; H, 3.96; I, 33.20. Found: C, 50.22; H, 4.07; I, 33.12.

(2-Iodoethynyl)(phenyl)iodonium triflate (14). To a cold $\left(0{ }^{\circ} \mathrm{C}\right)$ magnetically stirred solution of iodosobenzene diacetate (IBDA, $16.85 \mathrm{~g}, 52.3 \mathrm{mmol}$ ) in anhydrous $\mathrm{CH}_{2} \mathrm{Cl}_{2}$ (70 mL) under an Ar atmosphere, $\mathrm{CF}_{3} \mathrm{SO}_{3} \mathrm{H}(8.8 \mathrm{~mL}, 5.8 \mathrm{~g}, 99.4 \mathrm{mmol})$ was added dropwise. Then, the mixture was stirred at $0{ }^{\circ} \mathrm{C}$ for $30 \mathrm{~min}$ and 1-iodo-2-trimethylsilylacetylene (11.06 g, $49.4 \mathrm{mmol}$ ) was added dropwise and the solution was stirred at $0{ }^{\circ} \mathrm{C}$ for $2 \mathrm{~h}$, a white precipitate being formed. The solution was decanted from the solid, which was washed three times with anhydrous and cold $\mathrm{CH}_{2} \mathrm{Cl}_{2}$. The obtained light brown solid (16.35 g) showed to contain acetic acid (molar ratio $\mathrm{AcOH} / 14$ about 1:4 by ${ }^{1} \mathrm{H} \mathrm{NMR}$ ) and was crystallized at room temperature from a mixture of MeCN (8 mL) and $\mathrm{CH}_{2} \mathrm{Cl}_{2}(25 \mathrm{~mL})$ to give triflate $14(11.4 \mathrm{~g}, 46 \%)$ as a white solid. An analytical sample of $\mathbf{1 4}$ was obtained by repeating the above crystallization process, mp 96-98 ${ }^{\circ} \mathrm{C}$. IR (ATR): v 2106, 1561, 1469, 1446, 1293, 1272, 1212, 1170, 1019, 984, 730, 704, 672, 651, $631 \mathrm{~cm}^{-1} .{ }^{1} \mathrm{H}$ NMR (400 MHz, CD $\left.\mathrm{CDN}_{3}\right): \delta 7.61\left(\mathrm{~m}, 2 \mathrm{H}, \mathrm{Ar}-\mathrm{H}_{\text {meta }}\right), 7.78(\mathrm{tm}, J=7.4 \mathrm{~Hz}, 1 \mathrm{H}, \mathrm{Ar}-$ $\mathrm{H}_{\text {para }}$ ), 8.17 (dm, $J=8.8 \mathrm{~Hz}, 2 \mathrm{H}, \mathrm{Ar}-\mathrm{H}_{\text {ortho }}$ ). ${ }^{13} \mathrm{C}$ NMR (100.6 MHz, $\mathrm{CD}_{3} \mathrm{CN}$ ): $\delta 30.0$ (C, C1), 33.3 (C, C2), 117.7 (C, Ar-C1), $121.5\left(\mathrm{CF}_{3}, \mathrm{q}, J=320 \mathrm{~Hz}, \mathrm{CF}_{3} \mathrm{SO}_{3}{ }^{-}\right), 133.7$ [CH, Ar-C3(5)], 134.3 (CH, Ar-C4), 136.0 [CH, Ar-C2(6)]. HRMS (ESI-TOF): calcd. for $\left[\mathrm{C}_{9} \mathrm{H}_{5} \mathrm{~F}_{3} \mathrm{I}_{2} \mathrm{O}_{3} \mathrm{~S}-\right.$ $\left.\mathrm{CF}_{3} \mathrm{SO}_{3}\right]^{+}:$354.8475. Found: 354.8472. Anal. calcd. for $\mathrm{C}_{9} \mathrm{H}_{5} \mathrm{~F}_{3} \mathrm{I}_{2} \mathrm{O}_{3} \mathrm{~S}: \mathrm{C}, 21.45 ; \mathrm{H}, 1.00$; I, 50.36; F, 11.31. Found: C, 21.59; H, 0.95; I, 50.52; F, 11.51.

\section{Acknowledgements}

Financial support from Ministerio de Ciencia e Innovación (CTQ2008-03768) and Comissionat per a Universitats i Recerca (2005-SGR-00180) is gratefully acknowledged.

\section{References}

1. (a) Camps, P.; Fernández, J. A.; Font-Bardia, M.; Solans, X.; Vázquez, S. Tetrahedron 2005, 61, 3593 and references therein cited (b) Camps, P.; Muñoz, M. R.; Vázquez, S. J. Org. Chem. 2005, 70, 1945. (c) Vázquez, S.; Camps, P. Tetrahedron 2005, 61, 5147. (d) Camps, P.; Muñoz, M. R.; Vázquez, S. Tetrahedron 2006, 62, 7645. (e) Ayats, C.; Camps, P.; Fernández, J. A.; Vázquez, S. Chem. Eur. J. 2007, 13, 1522. (f) Camps, P.; Colet, G.; 
Delgado, S.; Muñoz, M. R.; Pericàs, M. A.; Solà, L.; Vázquez, S. Tetrahedron 2007, 63, 4669. (g) Camps, P.; Fernández, J. A.; Vázquez, S. Arkivoc 2010, IV, 74.

2. Teixeira, L. H. P.; Barreiro, E. J.; Fraga, C. A. M. Synth. Commun. 1997, 27, 3241.

3. Kumamoto, H.; Haraguchi, K.; Tanaka, H.; Nitanda, T.; Baba, M.; Dutschman, G. E.; Cheng, Y-C.; Kato, K. Nucleosides, Nucleotides and Nucleic Acids. 2005, 24, 73.

4. Kato, K.; Suzuki, H.; Tanaka, H.; Miyasaka, T.; Baba, M.; Yamaguchi, K.; Akita, H. Chem. Pharm. Bull. 1999, 47, 1256.

5. Luche, J. L.; Gemal, A. L. J. Am. Chem. Soc. 1979, 101, 5848.

6. Burger, U.; Erne-Zellweger, D. Mayeri, C. Helv. Chim. Acta 1987, 70, 587.

7. (a) De Lucchi, O.; Lucchini, V.; Pasquato, L.; Modena, G. J. Org. Chem. 1984, 49, 596. (b) Cossu, S.; Battaggia, S.; De Lucchi, O. J. Org. Chem. 1997, 62, 4162.

8. T.; Kotani, M.; Fujiwara, Y. Synthesis 1998, 1416.

9. Jahnke, E.; Weiss, J.; Neuhaus, S.; Hoheisel, T. N.; Frauenrath, H. Chem. Eur. J. 2009, 15, 388.

10. Williamson, B. L.; Stang, P. J.; Arif, A. M. J. Am. Chem. Soc. 1993, 115, 2590.

11. Stang, P. J.; Schwarz, A.; Blume, T.; Zhdankin, V. V. Tetrahedron Lett. 1992, 33, 6759.

12. Stang, P. J.; Zhdankin, V. V. J. Am. Chem. Soc. 1991, 113, 4571. 